\title{
DESIGNING AND IMPLEMENTING AN ARCHITECTURE WITH BOUNDARY SCAN
}

\section{R.P. VAN RIESSEN \\ H.G. KERKHOFF \\ A. KLOPPENBURG}

University of Twente, The Netherlands

The authors describe a standardized, structured test methodology based on the boundary-scan proposal from the Joint Test Action Group, which is now IEEE proposed standard P1 149.1. The architecture ensures testability of the hardware from the printed-circuit- board level down to integrated-circuit level. In addition, the architecture has built-in self-test at the IC level. The authors have implemented this design using a self-test compiler. he problems of testing increasingly complex digital integrated Isystems continue to challenge the design and test community. At the printed-circuit-board, or PCB, level, these problems led to the formation of JTAG, short for Joint Test Action Group, a collaborative organization of major semiconductor users in Europe and North America. JTAG subsequently developed the boundary-scan standard ${ }^{1}$ with the goal of improving the controllability and observability of an IC's primary inputs and outputs. Because of this standard, which is now IEEE proposed standard P1149.1, we can now easily implement testability hardware using computer tools, which reduces overall design time.

However, boundary scan does not address testability at the IC level-primarily because there is no standard for designing BIST circuits. At this level are many approaches to adding testability, but the one that seems most promising for future VLSI and ULSI circuits is built-in self-test, or BIST. ${ }^{2}$ In BIST, test data is generated and evaluated on the chip.

In this article, we present an architecture called hierarchical testable, or H-testable, architecture for integrating boundary scan at the PCB level and BIST at the IC level. We believe that this integration is important because the boundary-scan standard defines access to the IC during the IC test. The extra test pins let us control on-chip testability hardware.

A digital system has several levels of hierarchy. First, we have the PCB level, which contains such items as a Winchester control board. The second level is the IC level, where we have units such as a microprocessor chip. The third level, called the macro level, allows us to make finer distinctions between functional modules like PLAs and ALUs, for example-the so-called macros. ${ }^{3}$ We use these three levels to define the $\mathrm{H}$-testable architecture. With this hierarchical structure, we can use BIST for a macro at the higher levels and so more completely integrate the testability features at the IC and PCB levels.

As we show in more detail later, the $\mathrm{H}$-testable architecture can be implemented using a self-test compiler. ${ }^{4}$ This compiler automatically generates the layout of a macro, including hardware to generate data for and evaluate the results of self-test.

0740-7475/90/0002-09\$1.0001990 IEEE 


\section{Test interface elements are located at the primary inputs and outputs of both macros and ICs. Each TIE contains boundary-scan cells and serial control registers.}

\section{ARCHITECTURAL REQUIREMENTS}

To be H-testable, an architecture must have certain characteristics. First, it must be hierarchical because, as we just mentioned, the hierarchical approach allows us to use test results from a lower level in higher levels. The results of a macro test can be used for the IC test, for example. Second, the architecture has to be standard because PCB manufacturers use ICs from different vendors on a single board. If we have well-defined test-interface rules and control definitions for every level of hierarchy, we can use standard test approaches. Third, the architecture has to be structured also to reduce extra design time. With a structured approach, test hardware is developed only once and is reusable. A structured approach also facilitates the design by allowing us to use computer tools. Finally, to be $\mathrm{H}$-testable, the architecture must incorporate the BIST facilities of different macros.

\section{INTEGRATION WITH BOUNDARY SCAN}

Figure 1 shows the JTAG boundary-scan architecture for PCB testing. The behavior and architecture of all blocks in this figure are defined in the standard. ${ }^{1}$ We use this architecture to define our H-testable architecture.

Figure 2 is a schematic block diagram of the $\mathrm{H}$-testable architecture at the IC level. In this diagram, we can distinguish two levels of hierarchy: the macro level and the IC level. At the IC level are (self-)testable macros, connections between these macros, and additional testability hardware. The macro level consists of a (self-)testable macro with additional testability hardware. Both levels of the testability hardware incorporate test interface elements, or TIEs; a test processor; and a scan path.

The TIEs separate a macro (IC) from the connections with other macros (ICs). Therefore, TIEs are located at the primary inputs and primary outputs of both macros and ICs. Each TIE contains boundary-scan cells and serial control registers. Test processors provide parallel control of the TIEs.

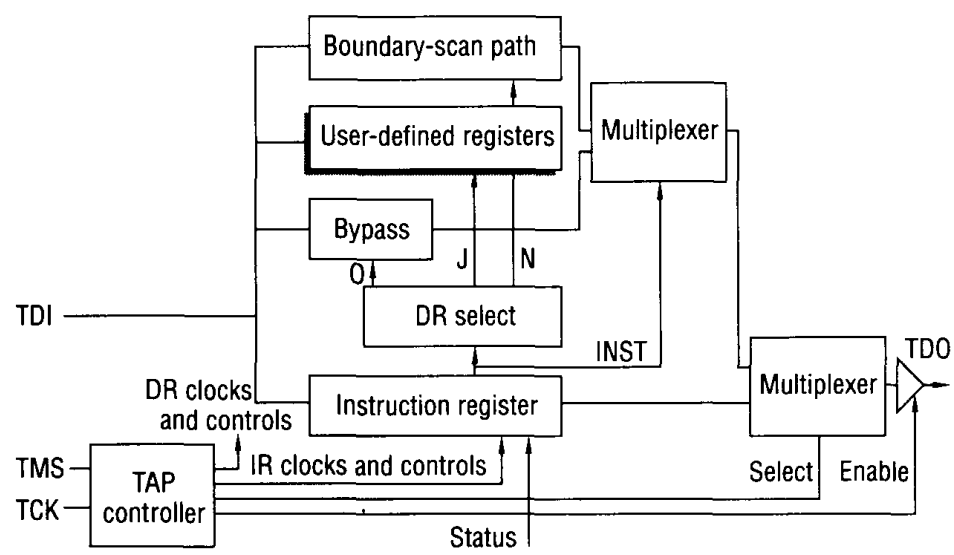

Figure 1. Architecture of the Joint Test Action Group boundary-scan standard. 
The IC test processor provides the TIEs at the IC level and the macro test processors with parallel control. The macro test processor provides the TIEs at the macro level with parallel control. The macro test processor can also control a macro self-test.

\section{ARCHITECTURE AT THE IC LEVEL}

At the IC level, the $\mathrm{H}$-testable architecture is compatible with the boundary-scan architecture and its behavior. Therefore, we have in effect merged the $\mathrm{H}$-testable architecture with the JTAG boundaryscan architecture as evidenced by the following structural characteristics:

- The JTAG boundary-scan path in Figure 1 is part of the boundaryscan cells of the TIEs at the IC's input and output in Figure 2.

- The JTAG instruction-register path is implemented in the IC-level test processor. The registers in this path provide the serial control data for the IC-level TIE.

- The JTAG test-access port (TAP) controller is implemented in the IC-level test processor of the $\mathrm{H}$-testable architecture. The TAP controller generates the parallel control signals for the IC-level TIEs and the macro-level test processors.

- The JTAG user-defined register path is used to implement the local scan path in Figure 2.

We can merge the architecture of the JTAG boundary-scan standard and the $\mathrm{H}$-testable architecture without any changes to either. Consequently, at the IC level, the H-testable architecture has already been defined.

\section{ARCHITECTURE AT THE MACRO LEVEL}

The test hardware for the H-testable architecture at the macro level consists of TIEs and a macro test processor, as Figure 2 shows.

A TIE in the local scan path forms the link between a macro and the macro interconnection. We add this element only to enhance testability. The TIEs are located at both inputs and outputs of a macro and do not affect the functional behavior of the IC during normal operation.
We can merge the JTAG

boundary-scan and

H-testable architectures

without any changes to either. Thus, the

H-testable architecture

is already defined at the

IC level.

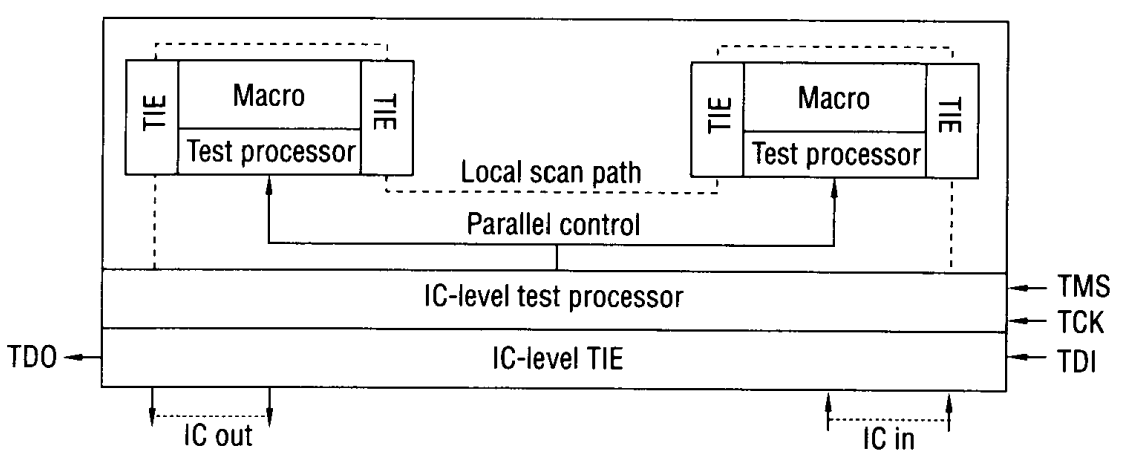

Figure 2. Block diagram of the hierarchical testable (H-testable) architecture at the IC level. 


\section{Data-register cells form the interface of the macro and macro interconnections. DRC modes vary according to the control signals applied to the multiplexers.}

During an IC test however, the TIEs are able to separate macros from their interconnections, which allows an independent test of both. Test patterns are shifted serially into the TIE via the local scan path, and the TIE applies the patterns in parallel to the macro or to the interconnection of macros. Results from a macro self-test are loaded in parallel into the TIEs at the output of the macro. Results from the macro interconnection test are loaded in parallel into the TIEs at the input of a macro. Next, data $m$ the TIE's will be shifted out serially via the local scan path. Control signals for the TIE are applied serially via the local scan path and in parallel via the control signals from the macro-level test processor.

Figure 3 shows the implementation of the TIE. A TIE consists of data-register cells $D$, two control-register cells ( $M$ and $S$ ), a bypass path, and a multiplexer.

Data-register cells form the interface of the macro and the interconnections to other macros. Figure $4 \mathrm{a}$ is a block diagram of one of these cells. This cell consists of two multiplexers and a master-slave register. The macro-level test processor provides the signals Mode. $\mathrm{DRC} 1$, and DRC2. The data-register cell is used in different modes, which vary according to the control signals applied to the multiplexers. Figure $4 b$ shows the truth table of multiplexer 2 . The first mode

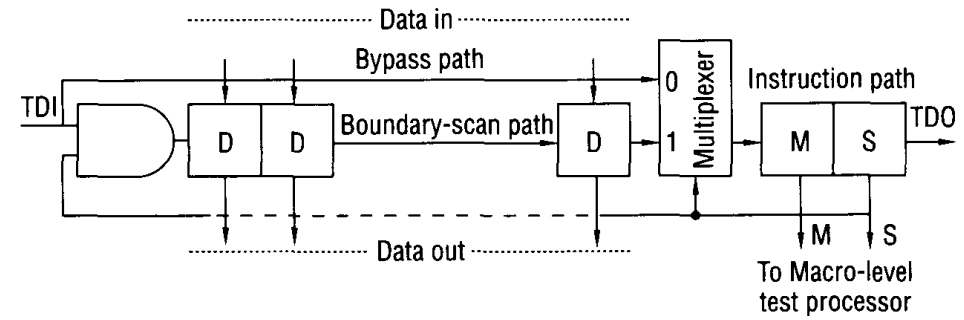

Figure 3. Implementation of a test interface element, or TIE; $D$ is the data-reg ister cell and $M$ and $S$ are control-register cells.
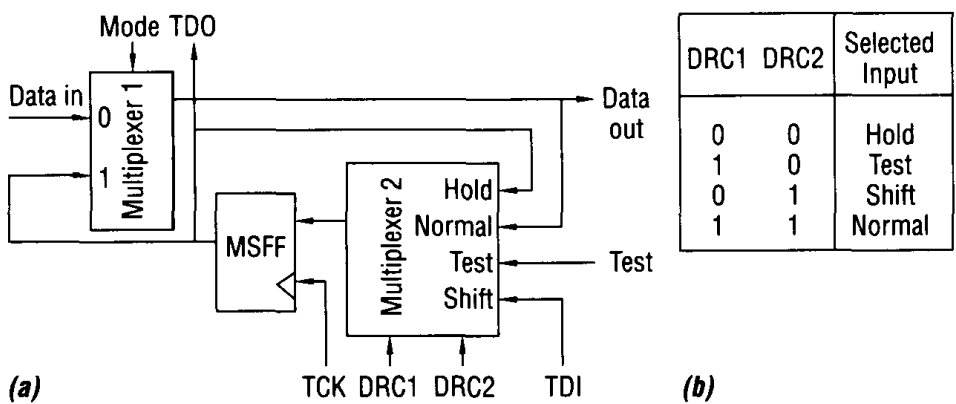

Figure 4. Block diagram of a data-register cell (a) and the truth table for data-register multiplexer 2 (b). 
of the data-register cell is the hold mode $(\mathrm{DRCl}=0, \mathrm{DRC} 2=0$ ), in which data in the register remains unchanged. The second mode (DRCl $1=1$, $\mathrm{DRC} 2=0$ ) is the test mode, in which the input Test is used for BIST. The third mode (DRC $1=0, \mathrm{DRC} 2=1$ ) is the scan mode, in which the cell is placed in the local scan path at the IC level. Figure 2 shows this path. We can now shift data into input TDI and towards output TDO. The fourth mode (DRC $1=1, \mathrm{DRC} 2=1$ ) is the normal functional operation. Data enters the cell via the input Data-In and propagates through the cell with minimal delay to Data-Out.

The control-register cells in the instruction path of a TIE ( $M$ and $S$ in Figure 3) provide its serial control. These registers consist of a shift register (L2) and an output latch (L1). Figure $5 \mathrm{a}$ is a block diagram of an instruction-register cell. The TAP controller of the IC-level test processor supplies the control signals Update-IR, IRC1, and IRC2. At the rising edge of Update-IR, the contents of shift register L2 are loaded into the output latch L1. The signals IRC 1 and IRC2 control which input is selected by the multiplexer. Figure $5 \mathrm{~b}$ shows the truth table of this multiplexer. The input Hold (IRC $1=0, \operatorname{IRC} 2=0$ ) is selected to retain the data in the output latch $\mathrm{L} 1$. The input Status $(\mathrm{IRC} 1=1$, IRC2 $=0$ ) is required to load a signal into the shift register. The input Shift $(\operatorname{IRC} 1=0 / 1, \operatorname{IRC} 2=1)$ is the serial scan input. This input is connected to the output TDO of the previous shift-register cell.

Because TIEs are at both the input and output of a macro, there are two mode registers-Ml at the input, $\mathrm{M} 2$ at the output-and two select registers-S1 at the input and S2 at the output. These four instruction registers can define 16 modes for the data-register cells.

The select register $S$ in Figure 3 controls the bypass of the dataregister cells. The data-register cells in a TIE are placed in the local scan path if $S=1$. If $S=0$ the scan path of a TIE contains only the instruction-register cells.

The value in the mode register $M$ is decoded in the macro-level test processor and, together with parallel control signals from the IC-level test processor, controls the two functions of the data-register cells. In Figure $4 a$ the data-register cells transmit data if mode $=1$ and receive data via input Data-In if mode $=0$.

Figure 3 shows, in contrast with the JTAG architecture, that the boundary-scan path and the instruction path are connected serially. With this architecture at the macro level, we can use a simple multiplexer to select either the bypass mode or the boundary-scan mode. Because both modes include the instruction path, a data scan will always contain data bits and instruction bits. We need only one scan operation to initialize the TIEs for a macro test. At the PCB level, the JTAG boundary-scan architecture requires two scan operations to initiate the TIEs. In the first stage, the instruction bits are shifted in. In the second stage, the data bits are shifted in.

Another difference between the macro-level TIE and the IC-level TIE is the number of modes that a data-register cell has. The boundaryscan data-register cell at the IC level has three modes of operation. At the macro level, it has four modes. As we mentioned earlier, this additional mode is the test mode, which allows the data-register cell to be used for BIST. This mode does not require an extra control signal as compared with the boundary-scan register cell.
At the PCB level, the JTAG boundary-scan architecture requires two scan operations to initiate the test interface elements: shift in instruction bits and shift in data bits.

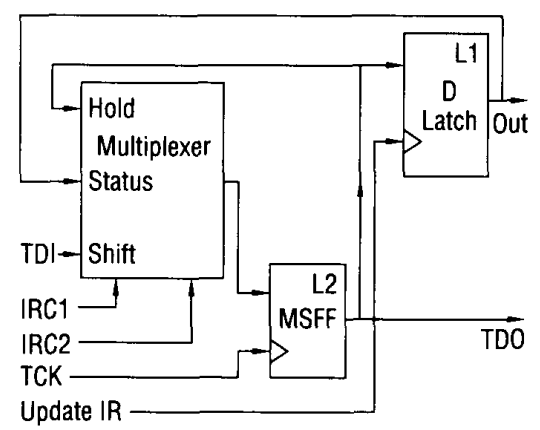

(a)

\begin{tabular}{|cc|c|}
\hline IRC1 & IRC2 & Selected Input \\
\hline 0 & 0 & Hold \\
1 & 0 & Status \\
0 & 1 & Shift \\
1 & 1 & Shift \\
\hline
\end{tabular}

(b)

Figure 5. Block diagram of an instruction register cell (a) and the truth table for the instruction-register multiplexer (b). 
Central to the

H-testable architecture

is the self-testable macro, which has only combinational logic.

\section{THE MACRO-LEVEL TEST PROCESSOR}

The test processor forms the control part of the H-testable architecture. At the macro level, the processor has to perform a macro self-test and apply the parallel control signals to the data-register cells of the TIEs at both the input and output of the macro.

To carry out BIST, we must generate test patterns and compact them using some hardware implementation of a test-pattern generation/compaction algorithm. Test patterns are applied in parallel to the macro inputs by loading the test patterns in the data-register cells via the extra Test input, as Figure 4a illustrates. The test result is loaded in parallel into the TIE at the output of the macro.

During the self-test, the macro test processor generates the parallel control signals for the data-register cells of the TIE. Figure 6 is

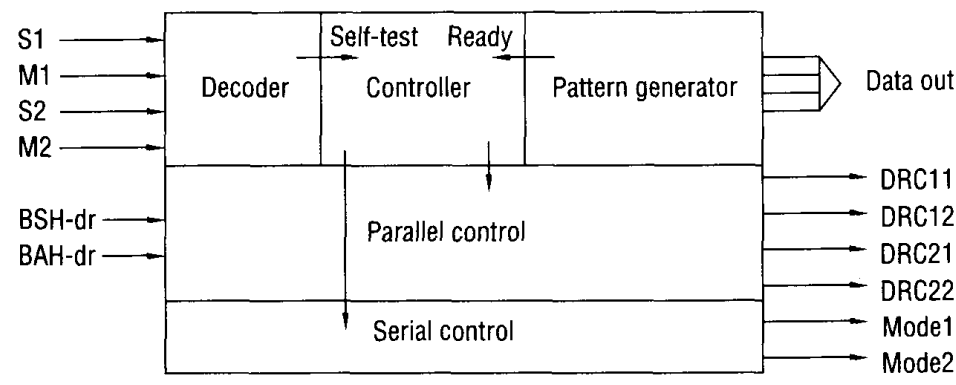

Figure 6. Block diagram of a macro-level test processor.

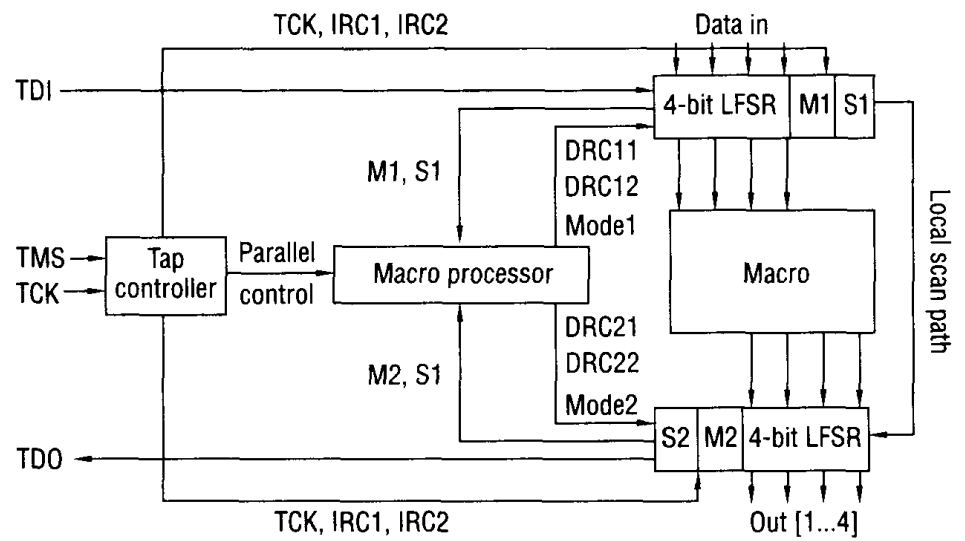

Figure 7. Example of the hierarchical testable, or H-testable architecture. 
a block diagram of the macro-level test processor. We briefly describe the main parts of the test-processor architecture. A more detailed description is available elsewhere. ${ }^{5}$

Parallel and serial control logic supply the data-register cells with control signals. The signals DRC11, DRC12, and Mode1 form the signals DRC1, DRC2, and Mode for the TIE at the input of a macro. The signals DRC21, DRC22, and Mode2 form the signals DRC1, DRC2, and Mode for the TIE at the output of a macro. These signals depend on the state of the controller and on the state of the IC-level TAP controller (BSH-dr, BAH-dr).

A decoder signals the controller to start a macro self-test. The self-test is activated by the contents of the registers in the instruction path at the input (S1 and M1) and the output (S2 and M2) of the macro. A controller, which is, in fact, a synchronous state machine controls the macro self-test. We can, however, implement a macro self-test in many ways, depending on the type of macro. Therefore, we have a dedicated controller for each macro. Every controller must be able to start the self-test, indicate the end of a self-test, and control the registers involved.

A pattern generator, which is governed by the controller, generates the test patterns for the macro. The pattern generator uses the TIE's data-register cells at the input of the macro. The generated patterns (Data-Out) are applied to the Test inputs of the data registers. The pattern generator generates a signal (Ready) for the controller to indicate the completion of a self-test.

\section{AN H-TESTABLE ARCHITECTURE}

The best way to illustrate the features of the $\mathrm{H}$-testable architecture is to describe an actual implementation. Figure 7 shows the example we have used. Our intent is primarily to show the integration of boundary-scan hardware with BIST at the IC level. Our example incorporates two TIEs, a macro test processor, one TAP controller, and a simple macro.

The central part in the architecture is the self-testable macro, which has four inputs and four outputs. This macro contains only combinational logic and is tested with pseudorandom patterns. A signature analyzer compacts the test results. We have added some hardware to the data-register cells of the TIEs so that we can use the data register as a building block for pseudorandom pattern generation and signature analysis. Figure 8 illustrates the additional hardware.

We form the pattern generator/compactor by connecting a number of modified data-register cells as a linear-feedback shift register. To do this, we feed Data-Out of the last register cell back to the Feedback terminal of specific data-register cells. The connections are determined by the feedback polynomial. ${ }^{6}$ We can use the structure as a pseudorandom pattern generator when $(\mathrm{DRC} 1, \mathrm{DRC} 2)=(1,0)$ and mode $=1$. The circuit operates as a signature analyzer when (DRC), $\mathrm{DRC} 2)=(1,0)$ and mode $=0$.

In our example, the TIEs form an LFSR during the test mode that has a feedback polynomial of $1+X+X^{4}$. Figure 9 shows the data register of a TIE realizing this LFSR.

The macro-level test processor also incorporates the logic to start and complete the self-test. The Ready signal, which indicates the completion of the self-test, is true when a specific test pattern is generated.
A pattern generator, which is governed by the controller, generates the test patterns for the macro. 


\section{We have added some hardware so that we can use the data register as a building block for pseudorandom pattern generation and signature analysis.}

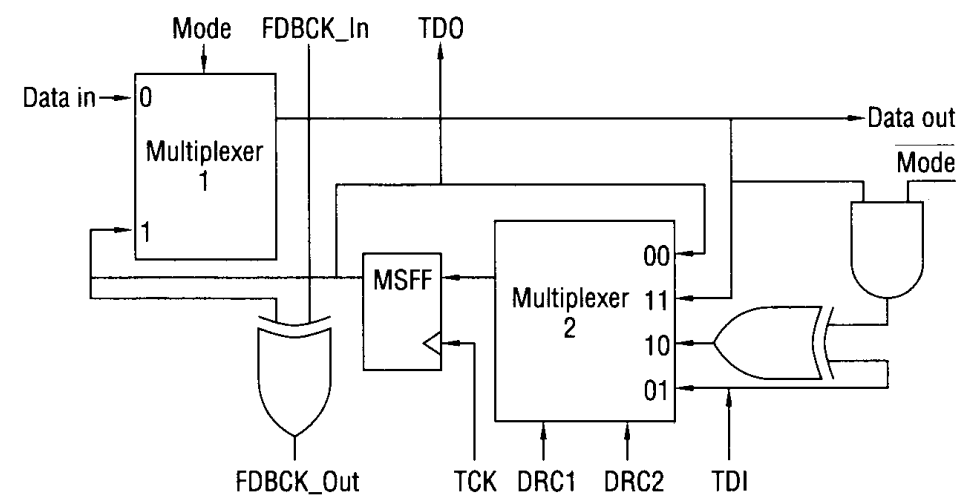

Figure 8. Block diagram of a modified data register.

Data in

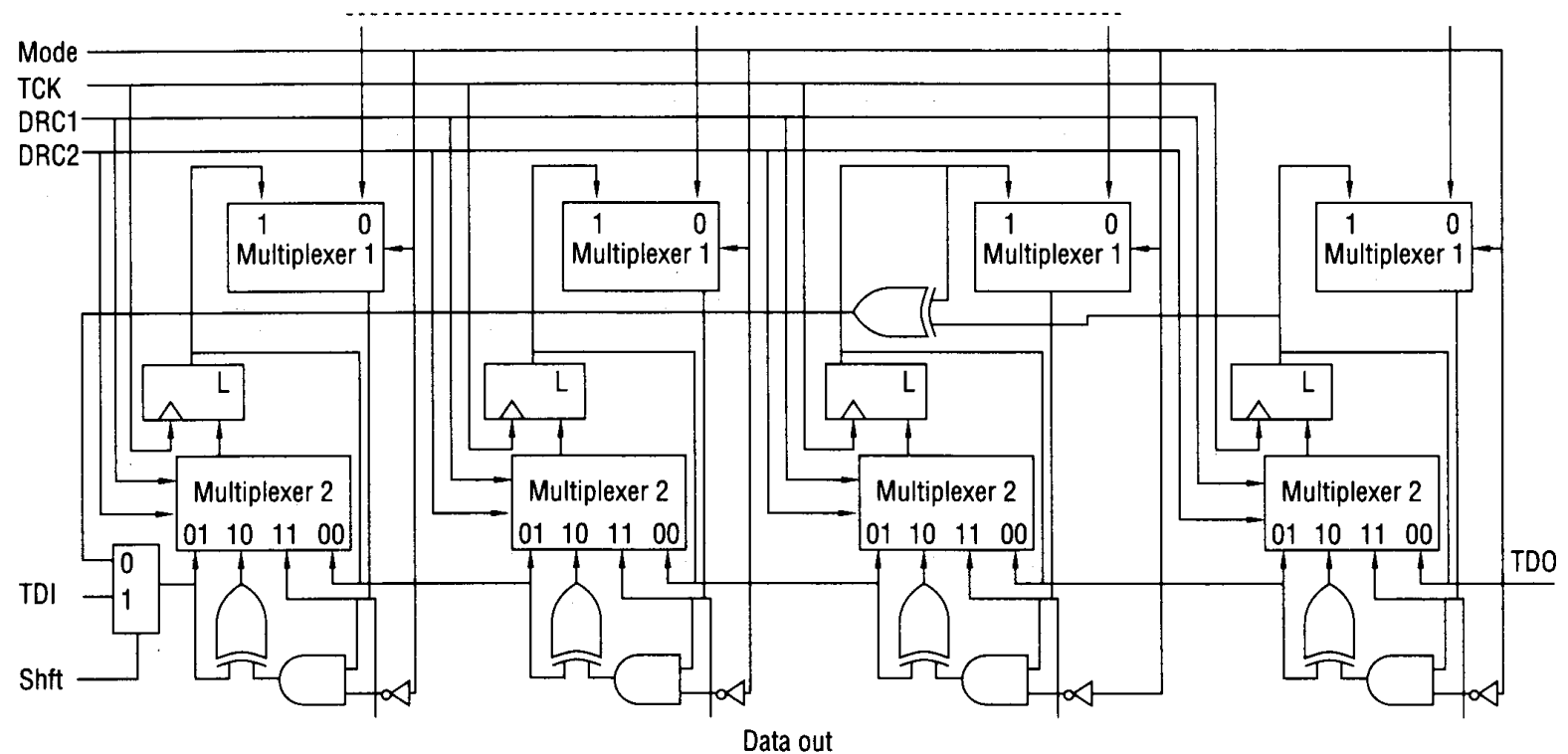

Figure 9. Block diagram of the data register part of the test interface element, or TIE, that forms a four-bit linear feedback shift register (LFSR) with feedback polynomial $1+X+X^{4}$. 
The TAP controller is identical to the TAP controller as described in version 2.0 of the boundary-scan standard. ${ }^{1}$

\section{SIMULATION OF THE SAMPLE CIRCUIT}

Table 1 shows the scan actions applied to the example circuit during simulation. Scan action 1 initializes the instruction-register cells. Four clock cycles shift the values for the initialization path into the instruction-register cells: $(\mathrm{M} 1, \mathrm{~S} 1, \mathrm{M} 2, \mathrm{~S} 2)=(1,1,0,1)$. Because $\mathrm{S} 1=1$ and $\mathrm{S} 2=1$, we can initialize the data-register cells at both the input and output.

Scan action 2 initializes the data-register cells for a macro self-test. Both data-register cells are initialized with the value $(1,1,0,0)$. Scan action 3 indicates that during a macro self-test, we can still shift data through the TIEs. While the two TIEs perform a macro self-test, a pattern is shifted via the input TDI to the output TDO.

We need scan action 4 to place the data-register cells in the scan path after the macro self-test has been completed. Finally, with scan action 5 , the signature in the output TIE appears at the serial output TDO.

We simulated the test process for this sample circuit using a switch-level description. ${ }^{7}$ The results of the simulation ${ }^{5}$ show the correct operation of the H-testable architecture. A layout for the individual blocks of the $\mathrm{H}$-testable architecture has since been designed and will be used in our self-test compiler.

\section{A SELF-TEST COMPILER}

As we mentioned earlier, the purpose of the H-testable architecture is to develop a standard, hierarchical test approach to ease the burden of test development. Towards that end, we implemented our architecture in a self-test compiler. ${ }^{4}$ The compiler automatically generates the layout of the most appropriate on-chip test hardware for self-testing along with the functional macro. Designers define only the type and size of the macro to be realized, along with the fault coverage they desire for the self-test. Using the described architecture, the compiler generates self-testable macros that we can control in a standardized format. The $\mathrm{H}$-testable architecture defines the signals to initialize, control, and verify a macro self-test from the macro level to the PCB level.

Table 1. Tests applied to the sample circuit in Figure 9.

\begin{tabular}{llcccc}
\hline \multirow{2}{*}{ No. } & \multicolumn{1}{c}{ Scan Action } & M1 & S1 & M2 & S2 \\
\hline 1 & Select initialization path & 1 & 1 & 0 & 1 \\
2 & Initialize data and instruction register & 1 & 0 & 0 & 0 \\
3 & Scan operation during self-test & 1 & 0 & 0 & 0 \\
4 & Select result path & 0 & 1 & 0 & 1 \\
5 & Verify test result and scan in & & & & \\
& pattern for external test & 0 & 1 & 1 & 1 \\
\hline
\end{tabular}

FEBRUARY 1990
With the self-test compiler, designers define only the type and size of the macro to be realized and the fault coverage they desire for the self-test. 
For small macros of say, 10 to $201 / O$ ports, the controllers will probably determine the overhead of the extra test hardware.
Figure 10 shows part of the layout of a self-testing carry-save array multiplier, which was generated by the self-test compiler. The selftest, performed in this particular structure, is an exhaustive test. We used a signature analyzer to evaluate the test responses. The bottom row of cells in the figure shows the layout of some data-register cells used for data compaction.

The overhead needed for the extra test hardware varies with the size of the array multiplier. For a $16 \times 16$-input carry-save array multiplier, for example, the overhead is about $20 \%$. For a $32 \times 32$-bit array multiplier, the overhead is about $12 \%$.

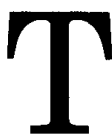

he $\mathrm{H}$-testable architecture we have described will ease the problems of testing ICs on printed-circuit boards. It is hierarchical, structured, and compatible with the JTAG boundary-scan standard for PCB testing. Using this architecture, we can initialize, control, and verify a macro self-test from the IC level up to the PCB level. During a macro self-test, the IC-level scan path can still be used, which implies that we can test different macros in

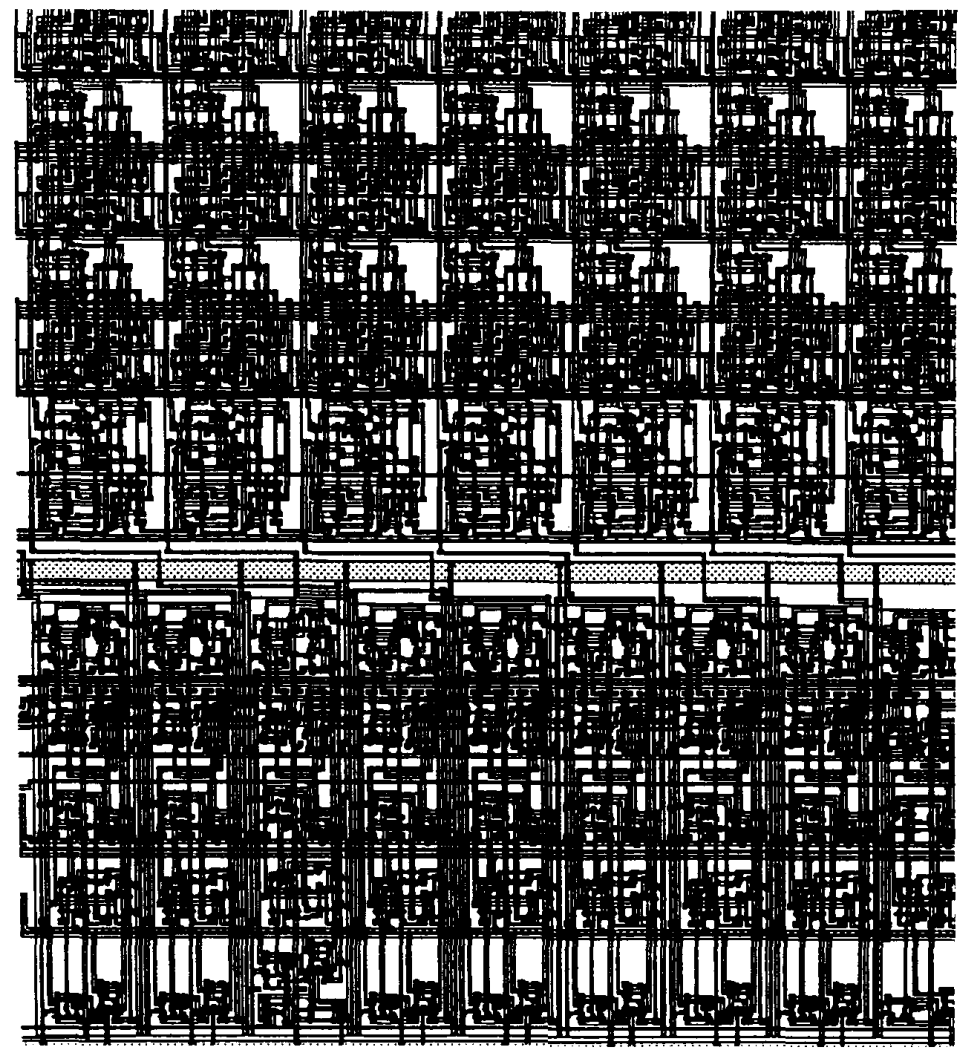

Figure 10. Part of the layout of a self-testing carry-save array multiplier, which was generated by the self-test compiler. The bottom row of cells shows the layout of some data-register cells used for data compaction.

IEEE DESIGN \& TEST OF COMPUTERS 
parallel with the H-testable architecture. We have implemented this architecture in a self-test compiler. An example circuit, generated by this compiler, shows the possibilities of this architecture.

The overhead of the extra test hardware remains a problem that needs more research. For small macros of say, 10 to $20 \mathrm{I} / \mathrm{O}$ ports, we expect the controller parts of the H-testable architecture to determine the overhead. Therefore, we advise the use of only one macro test processor for a set of small self-testable macros. 쇼맘

\section{ACKNOWLEDGMENTS}

This research is supported by the Innovative Research Program (IOP) on IC technology under HTO-049/1, part testing. We thank F. Beenker and his group at Philips Research Laboratories, Eindhoven, for their contribution to this research.
Ronald P. van Riessen is working towards a $\mathrm{PhD}$ in electrical engineering at the University of Twente, Enschede, The Netherlands, where his research interests include automatic design for testability and BIST of CMOS VLSI systems, particularly boundary scan with BIST in a module compiler. He holds an MSc in electrical engineering from the University of Twente.
Hans G. Kerkhoff is a staff member of the IC Technology and Electronics Group of the University of Twente, where his interests are in testable CAD for VISI digital signal processors. He holds an MSc in electronical engineering from the Technical University of Delft and $\mathrm{A} P \mathrm{Ph}$ in microelectronics design from the University of Twente.

\section{REFERENCES}

1. A Test Access Port and Boundary Scan Architecture, JTAG standard, Version 2.0, Draft 2, Jan. 1988.

2. B. Koenemann, "Built-In Logic Block Observation Techniques," Proc. Int'l Test Conf., 1979, pp. 37-41.

3. F. Beenker et al., "Macro Testing: Unifying IC and Board Test," IEEE Design \& Test of Computers, Vol. 4, No. 6, Dec. 1986, pp. 26-32.

4. R. van Riessen, H. Kerkhoff and A. Kloppenburg, "Design of a Compiler for the Generation of Self-Testable Macros," Proc. European Solid-State Circuits Conf., 1988, pp. 194-197.

5. A. Kloppenburg, Investigations on the Possibilities of Using Boundary-Scan Techniques in Silicon, MSc thesis, Univ. of Twente, 060-7001, Enschede, The Netherlands, July 1988.

6. A. Miczo, Digital Logic Testing and Simulation, John Wiley \& Sons, New York, 1987.

7. A. van Genderen, A. de Graaf, "SLS: A Switch-Level Timing Simulator," in The Integrated Design Book, Delft Univ. Press, 1986, The Netherlands, pp. 2.932.145 .

irect questions or comments on this article to $R$. van Riessen, University of Twente, EF Bldg., PO Box 217, 7500 AE Enschede, The Netherlands.

Koppenburg is a staff engineer at Witteveen en Bos, an engineering consultancy in Deventer. The Netherlands. He holds an MSc in electrical engineering from the University of Twente.

FEBRUARY 1990 